\title{
Adaptive analysis \& reconstruction of 3D DICOM images using enhancement based SBIR algorithm over MRI.
}

\author{
Padmaja Grandhe ${ }^{1 *}$, Sreenivas Reddy Edara ${ }^{2}$, Vasumathi Devara ${ }^{3}$ \\ ${ }^{1}$ Research Scholar, CSE Department, JNTUK, Kakinada, A.P, India \\ ${ }^{2}$ Principal, ANU College of Engineering, Guntur, India \\ ${ }^{3}$ Professor, CSE Department, JNTUH, Hyderabad, India
}

\begin{abstract}
The images can be visualized in three dimensional (3D) by means of different technique, these 3D techniques are used to improve the view of images. Converting two dimensional (2D) images into 3D images is a significant part of image application. A resourceful approach of 3D image reconstruction is implementing to execute a certain procedure that applied to $\mathrm{X}$-ray medical image. The paper deals with the DICOM images reconstruction which is originally very helpful for manufacture of modified anatomical implant by using rapid prototyping technology. The researchers are gradually focusing on biomedical 3Dimaging improvement. The MAT LAB image processing has been powerfully developed and almost implements in every modern topographical modalities. The CT slices of involved region of modified implant in DICOM arrangement are initially pre-processed using developed MATLAB code. These files can be additional used for Rapid Prototyping. This project in biomechanical area has been urbanized to give the ability in very cheap cost with highest technological support moderately with expensive import facilities and software. This approach is implemented via four steps preprocessing, image enhancement, image contour then image reconstruction and visualization. Suggest a proposed researcher is based on novel analytical model which can put to gather in sequence from any source (i.e.) Slicer Based Image Retrieval (SBIR) mechanism to get back applicable medical imagery bottom on their features.
\end{abstract}

Keywords: Slicer based image retrieval (SBIR), MRI, Bio medical image, Textural data, 3D imaging.

\section{Introduction}

Digital Image processing [1] is an emerging field in which doctors and surgeons are receiving dissimilar easy pathway for the analysis of composite disease such as cancer, brain tumor, breast cancer, kidney stones, etc. The detection of brain disease $[2,3]$ is a very demanding task, in which particular care is taken for image segmentation. An exacting part of body is scanned in the discus applications of the image analysis and techniques such as MRI $[2,4], \mathrm{CT}$ scan, and X rays. The images are judge by physician or surgeons to solve the problems. Brain analysis is a big cause of disability and death worldwide and connected abnormalities comprise for major changes in life. A tremendous enlargement has been done in the last decade for intelligence tumor in the area of intellectual cancer diagnosis. Cerebral cancer [5] has been notice that is dispersal over the world and many colleges and academy medical research centers are focusing on the issue [6].

The biomedical imaging at the present time is more focuses on the capture the images procedure for the therapeutic and diagnostic. The biomedical imaging technology is rarely used to use the enduring limb such as ultrasound, MRI, CT scan, and light endoscopy. Biomedical imaging used to right of admission the organ state in the body or the issues in the body. Today the software that used to do biomedical imaging reinverted since the X-ray was inverted on year 1895. The modem x-ray nowadays just need milliseconds of contact time and used the solids state electronics to produce a high quality images and resolution [7].

In image enhancement, the objective is to emphasize certain image features for following analysis or for image display. Examples comprise difference and edge enhancement, pseudo coloring, noise filtering, sharpening and magnifying. Image enhancement is helpful in feature removal, image psycho analysis and visual in order display. The enhancement procedure itself does not increase the inherent in order display in the data. It simply emphasizes sure particular image individuality. Enhancement algorithms are usually interactive and request dependent. Medical image analytic equipments such as CT scanners, MRI, ultrasound etc. have been used worldwide. The output of these equipments is useful for manufacture of modified anatomical implants. But this is likely 
only at some high quality hospitals and medical diagnostics centers. These equipments have been on the other hand assist by in order technology, which need strong computers with devoted software's for maintain patients specific data $[2,4]$. The initial data necessary for modified implant is taken from locally obtainable low grade medical scanners $[2,4]$.

Many researchers are developed 3D image retrieval methods. In this technique, the form of the 3D model is cautious as an elastic solid, with a simplex-mesh (i.e. limited component triangulation) exterior made of thousands of vertices. Deformations of the copy are resultant from a linear system that encodes exterior armed forces from the border of a Region of Interest (ROI). The prospect move in the direction of its base on the shout of the low decree payment picture and the resultant high occurrence sub-band images obtain by Discrete Wavelet Transform (DWT). New results on both 2D and 3D images show how our technique enhances the image's particulars and conserve edges [2,8].

\section{Related work}

Yavariabdi et al. [9] proposed studies 2D and 3D wavelet area medical image declaration improvement method. The proposed move toward is based on the exclamation of the low resolution input image and the resulting high frequency sub-band images obtain using Discrete Wavelet Transform (DWT). Experimental results on both 2D and 3D images show how our method enhances the image's details and preserves edges. In the last decades, many researchers have attempted to improve medical images and expand methods to reconstruct 3D image [4]. Proposed an included tool for the discovery and assessment of orthopedic fractures in long bone digital X-ray image. It is helpful to obtain $3 \mathrm{D}$ images for appraisal of patient case and diagnose the disease in many cases such as cancer diseases on vessel morphology and tissues described in $[3,5]$ obtainable a appraisal of the state-of-the-art approach on reconstruction of high-contrast coronary arteries from X-ray angiography. They also discuss the possible role of reconstruction in clinical decision making and interventional guidance, and highlight areas for future research.

Hama and Al-Ani [10] proposed a new idea in digital medical images are frequently exaggerated by surplus noise, haziness and suffer from lack of difference and unevenness which from time to time results in false analysis. Main target of this paper is to procedure a medical image so that the result is more appropriate than the original image for a medical analysis. This is achieved by apply a well-organized move toward for an adaptive anisotropic dispersal algorithm. In this paper a color images and medical images are improved using a new edgestopping purpose for an efficient adaptive anisotropic diffusion algorithm to improve the presentation of the well-organized adaptive anisotropic diffusion filter [11]. Proposed an approach to $3 \mathrm{D}$ rebuilding of brain tumor and opinion of its quantity from a set of two dimensional (2D) cross sectional magnetic resonance (MR) images of the brain. They do the work by rising methods for segmentation, inter-slice interpolation, mesh generation and generalization. A 2D/3D mail building method based on a non-rigid 2D point matching process is presented by idea et al. [12].

Radhika [13] proposed improving the clinical capability medical image improvement plays very important role for examination of image. To gain the correctness of an image enhancements method is used. The extra in order which is gain by improvement is used for medical analysis and analysis. This method improves the quality of that image and conserves the correctness. In image improvement the result is more able to be seen and appropriate than the original image for exact information. When images are improved for human viewers the object may be to get better the image quality, intelligibility for visual appearance.

\section{Proposed Overview Slicer Based Image Retrieval (SBIR) In 3D over Medical Environment}

SBIR histogram equalization is an advanced enhancement technique in the image processing which uses the retrieve the flow from database of image. Segmentation of image plays a vital role in a lot of medical imaging application by automatically locate the region of interest. Segmentation of image is the more vital function in figure analysis and processing. The segmentation results of a shape affect all the following processes of image analysis. This is essential to expand medical figure segmentation algorithms that are precise and well-organized (Figure 1).

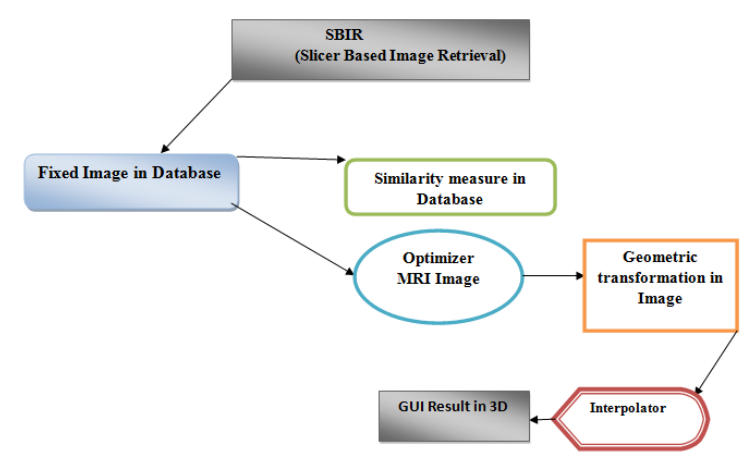

Figure 1. Proposed methodology flow diagram.

In this work; develop a dualistic sub-image histogram equalization based enhancement and segmentation techniques. Dualistic Slicer based Image Retrieval (DSBIR) which divides the image histogram into two parts base on the contribution mean and median in that order then equalizes each sub histogram separately. Further in the direction of enhance work; use GUI to present better result as compared to previous work. The proposed method has been experienced and evaluate on several medical images. In paper, the medical figure is lineated and extract out so that it can be viewed individually. Results show that the developed algorithm is highly well-organized over hierarchical grouping technique.

Let the Region of Interest is represented with ROI, Fixed Image Database with FID, 3D feature based parameter with $\mathrm{R}$ 
and Vector in Magnitude is represented with $\mathrm{V}$ in the study. The researcher focus at obtainable in the region of the interest (ROI) MRI image are used. On the other hand, it is classified as database image, if only a part of the slicer view in the ROI is used. Typically, the $3 \mathrm{D}$ image concentration base method is inclusive and the feature based methods are limited. An ordinary medical image, set in the database to get back the image can be distinct as a function FID (Fixed image database) that is, $\mathrm{R}$ is distinct in a separation of a three dimensional space and has principles in $\mathrm{V}$. However, in a number of imaging modalities, like Dispersal tensor magnet resonance imaging (DT-MRI), the picture can have principles in a multidimensional space $[14,15]$.

\section{Overview AVR technique in DICOM 3D analysis}

Advance technique is implemented Image fusion is the procedure that combine in order in multiple images of the same scene. This imagery may be captured from different sensors, acquire at different times, or have dissimilar spatial and spectral characteristics. The object of the image fusion is to retain the majority attractive individuality of each image [16]. With the ease of use of multisensory data in many fields, image fusion SBIR has been receiving rising notice in the researches for a wide spectrum of applications [1]. Here we use SBIR image fusion algorithm based on 3D transform which faster developed was a multi resolution analysis image fusion [14].

Before one can do image analysis and quantification, and request must faultlessly be able to right of entry, read and write image datasets in industry-standard formats. Conformance to conventional standards (i.e. DICOM, TIFF etc.) ensure compatibility with there and future application and checkup equipment. This in turn protects the researcher's investment in hardware and provides flexibility in reaching their goals.

\section{Proposed algorithm proposed algorithm}

- Let I be the input tumor image

- $\quad \mathrm{I}=$ add_noise (I, noisy type)

- $\quad$ I=remove _ noise (I, Filter type)

- $[\mathrm{m}, \mathrm{n}]=$ image segmentation (I, threshold value)

- $\mathrm{KL}=$ feature Extraction (I, threshold value)

- Train 1=SVM-classifier ( KL);

- $\quad$ Result=Train 1

- Result is tumor or non tumor segmented a particular boundary image.

The algorithm that we have proposed is as follows:

- Give MRI image of brain as input.

- Convert it to gray scale image.

- Be relevant high pass strain for image noise removal.

- Affect median filter to improve the quality of image.

- Compute threshold segmentation.

- Calculate divide segmentation.

- Figure morphological Process.

- Finally output will be a multi-tumor region
1. Let $\mathrm{x} 1 \ldots \mathrm{X} \mathrm{M}$ are $\mathrm{N}$ data points in the input image, let $\mathrm{k}$ be the number of region which is given by the user.

2. Choose R1.... rk region of centers.

3. Distance between each pixel and each cluster centre is found.

4. The distance function is given by

$J=\left|X_{i}-R_{j}\right|$ for $i=1, \ldots, N$ and for $j=1, \ldots, k$, where $\left|X_{k}-R_{k}\right|$, the absolute difference of the detachment among an information tip and the cluster centre indicates the distance of the $\mathrm{N}$ data points from their respective cluster centers.

Distribute the data points $\mathrm{x}$ among the $\mathrm{k}$ clusters using the relation

$X R_{i}$ if $\left|x-R_{j}\right|<\left|X-R_{k}\right|$ for $i=1,2 \ldots . . R$ where denotes the set of data points whose Region cluster centre.

Repeat from an initial to Step till meeting is meet. Following segmentation and discovery of the preferred area, there are chances for miss clustered regions to occur after the segmentation algorithm, hence morphological filtering is performed for enhancement of the tumor detected portion. Here structuring element used is disk shaped [14].

\section{Proposed Algorithm for 3D Images Analyzer}

The 3D units calculated in MRI are the rectangular prisms that are shaped by the pixels as $\mathrm{x}$ the bottom and the part thickness as the height. In order to improve the cross-section images, it be meant to get interference-free images by using image processing techniques. Among this process, dissimilar filter method was used in order to decide the limits that were the essential obligation for 3D modeling. Image dispensation consists of stages of contour processes, doorsill, segmentations, and filters. In the stage of contour processes; contours of any of current medical images were taken (i.e., removing its frames). In the threshold stage, value of any image was obtained. The SBIR algorithm possible edges of the substance with complex limits are determined by edge strength of mind methods (Figure 2). During this process, way of every likely edge is also strong-minded. As the second step, the likely edge change is joint by using the way information and the final rank of the edges is obtained. Basic principle of edge searching operators is based on the comparison of the brightness values between every pixel in the image and its neighbors $[1,16,17]$.

As already confirmed, smooth viewing cause by interruption technique create a serious difficulty on edges. Hence, preserve edges have to consequence in improved production images. The trouble in existing contribution researcher analyses of the 2D proposed algorithm. In order to relate the proposed algorithm to 3D images, the 3D SBIM algorithm has been selected to protect the edges and smooth viewer in GUI $[15,17]$. The high frequency sub-bands such as MMM, MML, MLM, LMM, LML, LLM, and MLL (where M and L are Maximum and Low coefficients) contain the edges of the low resolution image [18]. In addition, the size of Maximum frequency mechanism of ADWT (Adaptive Wavelet 
Transform) is increased by $3 \mathrm{D}$ bi cubic exclamation with factor $2[19]$.

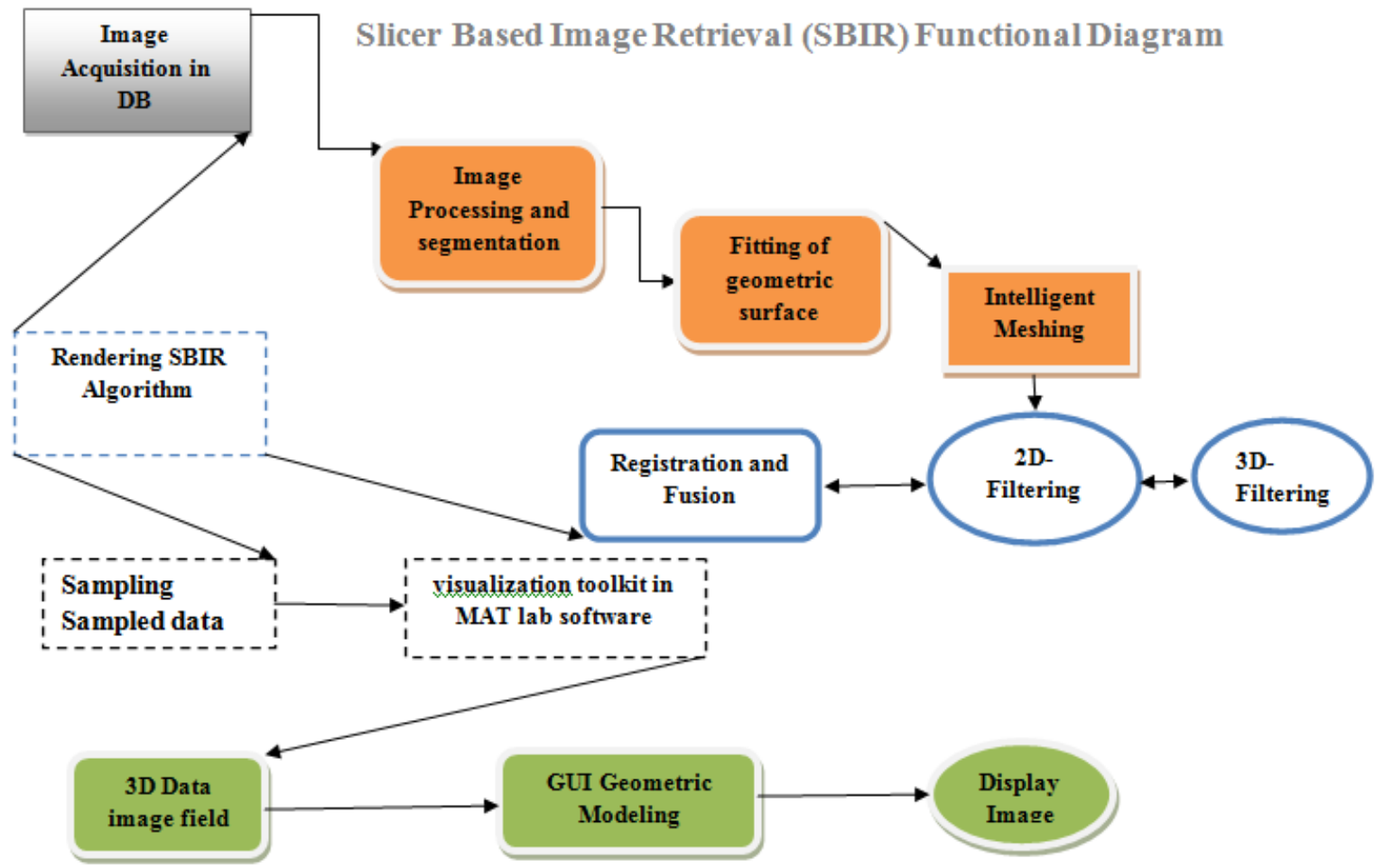

Figure 2. Slicer based image retrieval (SBIR) functional diagram.

The researcher goal of this learns was to assess the usefulness of 3D Slicer for segmentation of MRI compare to physical slice-by-slice user interface. We used two metrics for this assessment:

a) The time it took for physician to section MRI physically $v s$. by $3 \mathrm{D}$ Slicer,

b) The conformity among the two segmentations [14,20-22]. In by these metrics to assess our results, our supposition is that if 3D Slicer can be used to create segmentations that are statistically equal to the physicians attain manually, and in considerably less time, then the tool is helpful for volumetric follow-ups of MRI images [11].

\section{Image acquisition in data base}

The amplified significance of automatic computer method for anatomical brain mapping from MR images and quantitative brain image test method leads to a better than previous for validation and appraisal of the consequence of image on appearance of these events [23].

\section{Image processing and segmentation}

This researcher present an algorithm for medical 3D image de noising and segmentation by superfluous discrete wavelet transform. First, present a two stage denoising algorithm using the image synthesis concept. The algorithm starts with internationally de noising the brain images (3D volume) and
SBIR based algorithms follow by combine the outputs using entropy based fusion move toward [24,25].

\section{Fitting of geometric surface}

Geometric surface is analysed in MRI image in slicer view as per mouse movement in zoom in and zoom out operation is possible [2].

\section{Rendering SBIR algorithm}

SBIR fully based slicer in ROI image retrieval in different shape in $\mathrm{X} \& \mathrm{Y}$ automatic segment analysis. The method of MRI surface representation is that, first arrangement out of the center (such as surface, plane etc.) and then by the customary computer graphics techniques such as model, surface mapping method to attain the picture drawing [2]. Surface rendering is the most famous of the advance marching cubes algorithm $[26,27]$.

\section{Registration and fusion}

Individual internal-feature point was sort to unite with preselected external-feature point for corresponding procedure from side to side 3D-reconstruction and virtual-dissection. By following the events of feature-extraction and image-mapping, the process of option point to form plane and picking planes for segment were executed $[16,28]$. 


\section{D-filtering and 3D-filtering}

From side to side a two-dimensional filter can decrease the representation noise property, improving the sign to noise ratio and the removal of image's wake (Table 1). Take the medicine MRI image interruption method, the key part of isotropic dispensation, right of entry to data after three filters, dissimilar tissues and organs need segmentation and categorization, on the same site of the dissimilar image register and fusion, in order to make easy additional to a region of interest of the process $[15,29]$.

Table 1. AHOG (Advance histogram gradient value).

\begin{tabular}{lll}
\hline Features & Symptomatic & Symptomatic \\
\hline Skewness & 1.191 & 0.3409 \\
\hline Kurtosis & 9.7398 & 2.167 \\
\hline Entropy & 15.9552 & 21.1875 \\
\hline Joint entropy & 4.3539 & 8.1141 \\
\hline
\end{tabular}

\section{D Medical Image Visualization Technology in Simulation Environment}

MRI Medical image apparition is the alteration from MRI and other digital imaging knowledge to attain the in order of human body visually on the computer presentation for the threedimensional result, so as to give with custom all method to get in order. In the medicine field, the in arrange of is get by MRI and other digital imaging knowledge which to be visually on the computer appearance for the $3 \mathrm{D}$ effect $[2,23,30]$.

\section{Advance magnetic resonance imaging (AMRI)}

It provides so good difference flanked by soft tissues and is well-suited for imaging the brain and other tissue with high proton compactness. The two gaining series yield high and near-isotropic representation resolution. The pulse series (e.g., T1/T2-weighted) second-hand can be tune to attain best tissue contrast. As a result, 3D feel psychoanalysis in MRI has been the majority widely deliberate $[18,19]$.

\section{Flow Chart for Slicer View in 3D}

The Figure 3 is the MRI Slicer View in 3D is obtained by this flow chart work mechanism in segmentation process starts with the initialization of the advanced grow cut algorithm by the client on an axial and coronal slice. Then, the routine slicer $\mathrm{X} \& \mathrm{Y}$ view is in progress and afterwards review by the client $[4,8,11]$. These consequences addicted to the modification stage where the mat lab tools under Slicer are used to right the automatic segmentation consequence. Typically by navigate the length of the axial slices. Throughout the assessment stage the time for the initialization and the modification has been deliberate. The overall workflow of the future study is accessible on the right side so, the information is alienated into different data sets the preparation data set and the assessment data set. However, for the estimate stage additional image processing volume calculation is necessary to work out the Comparison Coefficient (CC) and the Distance (D) for a quantitative evaluation $[11,30]$.

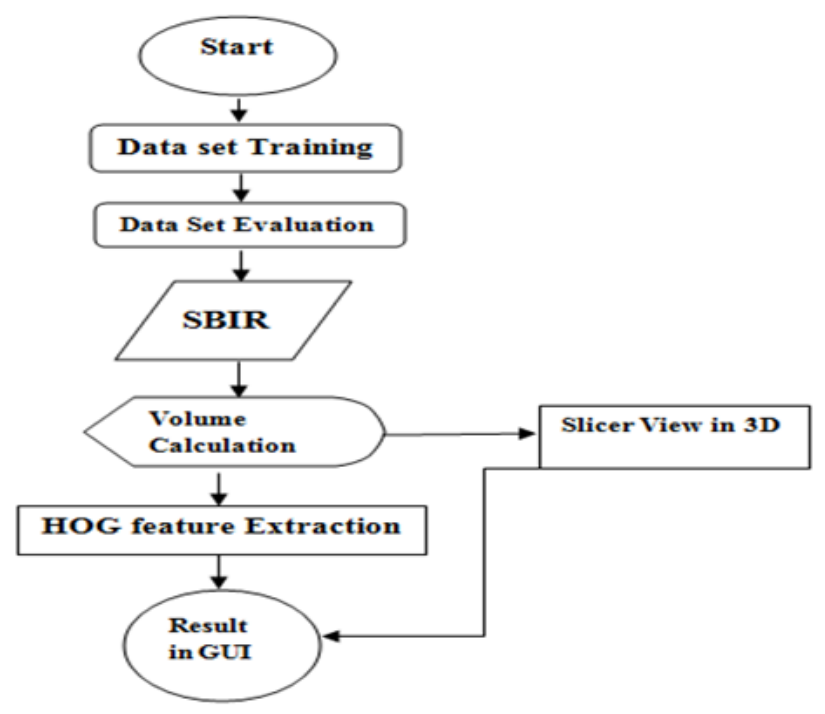

Figure 3. Flow chart.

\section{Implementation and Result Analysis}

Present the presentation of AMRI image retrieval using separate slicer based image retrieval and 3Dimensional analysis in slices view distance method. First in attendance the consequences of image retrieval using the method on a data set to assess its strength and exactness. Then present the contrast of dissimilar image in database with two dissimilar formulations X \& Y. Here GUI is shaped to examine the shock of the future method as shown in Figures 4 and 5 comparison measures like, remember and accuracy is intended for planned algorithm and compare with other method depict in Figure 6 [15].

The projected technique is discussed and contrast with additional dissimilar MRI decree development techniques. 2D slices of 3D volumetric MRI images and 3D MRI images Figure 4 shows the high resolution images were down sampled by a factor of 4 to create low resolution images [18]. In order to put into practice this $3 \mathrm{D}$ brain and interior brain structure segmentation in MR images professionally, mat lab program is used. This agenda could pace up the growth of this scheme since it has services to illustrate forms and to add records easily the future technique was practical to 10 clinical MRI datasets of various and types.

\section{Real time MRI database in slicer view}

Demonstrate the results database on different cases, Figure 4 shows that, which manual segmentation of more than a few shapes was obtainable, and which show 3D with dissimilar shapes, location, sizes, intensities and contrasts. Assessment of the segmentation consequences was performing from beginning to end quantitative comparison with physical segmentations, using quantity and outside measures. 
Segmentation consequences are quantitative evaluation are high accurateness.

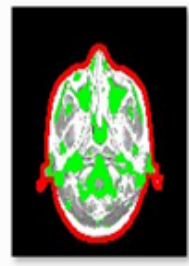

mriovr-000

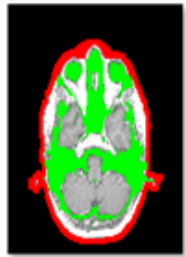

mriOvr-004

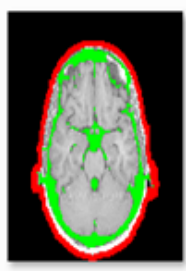

mriOvr-008

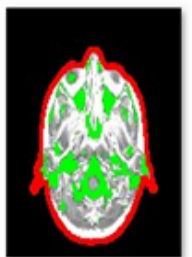

mriOvr-001

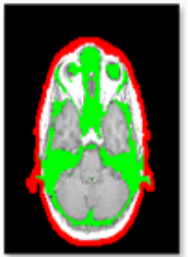

mriOvr-005

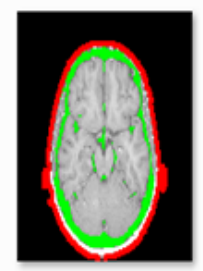

mriOvr-009

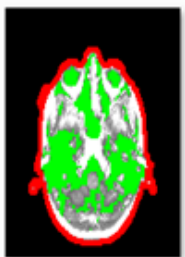

mriOvr-002

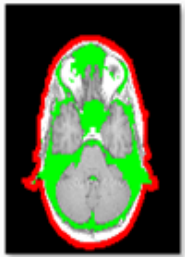

mriovr-006

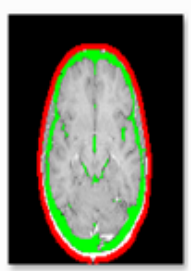

mriovr-010

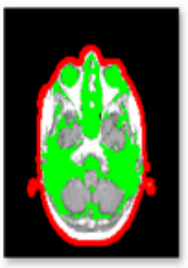

mriOvr-003

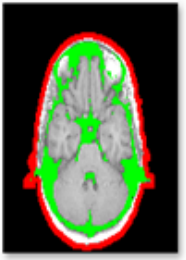

mriOvr-007

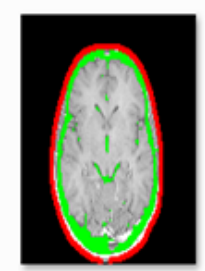

mriOvr-011
Figure 4. Real time MRI database in slicer view.

\section{Selection of image database ROI base $3 D$ viewer}

The method of selection of image database ROI base is shown in Figure 5.

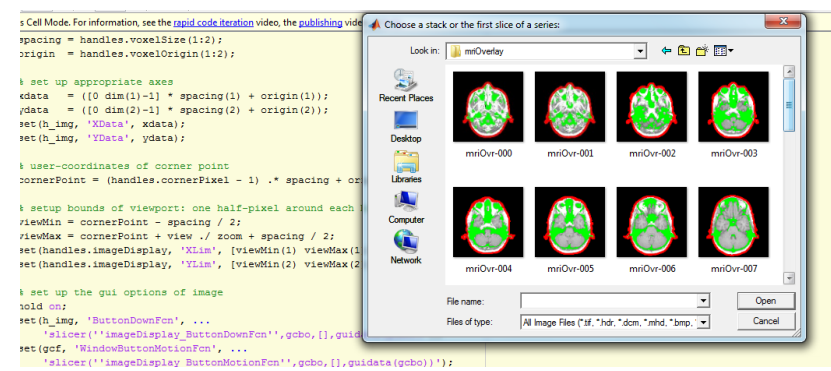

Figure 5. Selection of image database ROI base 3D viewer.

\section{Simulation library}

Need to install the all kind of module in simulation environment to execute the pre \& post processing mechanism.

\section{Graphical user interface brain MRI image}

The replica creator is used to make 3D outside model from label representation data as shown in Figure 7. The MRI slicer view represent the maker is a line of algorithms that create from the contribution tag map generate an advance marching cubes model (AMCM), runs triangle decrease and triangle smooth algorithms. The ROI Slicer was optimized for $1 \mathrm{~mm}$ brain MRI data. No decimation is practical however the default value of decimation for $3 \mathrm{D}$ slicer is 0.25 .

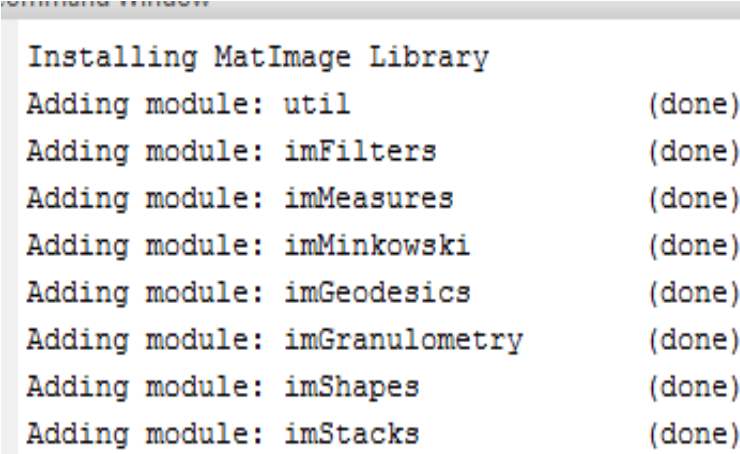

Figure 6. Installing mat image in command window.

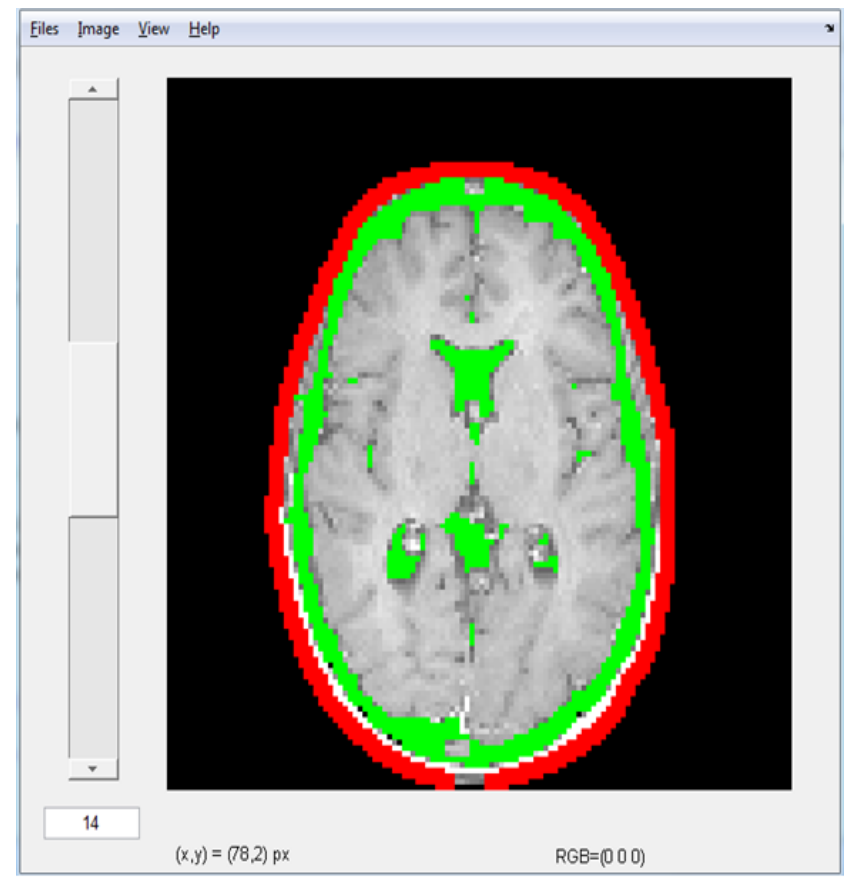

Figure 7. MRI slicer view.

\section{Slicer variation in $X$ \& $Y$ position}

These X \& Y models show the loaded in the model module for additional dispensation (Figure 8). This component is secondhand for loading, saving, altering the look of, and organize $3 \mathrm{~d}$ outside models. Model hierarchies let you to collection jointly model. When using the position changer Maker module, multiple dimensional is shaped from one tag map are group under a replica pecking order node and as a result we can have a slicer MRI image.

\section{SBIR slices MRI 3D view}

A Slicer Based Image retrieval Hierarchy Tree is prearranged which allow create ortho-slices in GUI nodes, drag and drop models control color and visibility of models and hierarchies, search, delete, and rename MRI image (Figure 9). The replica info tab keeps the documentation of the capacity in use as the representation is life form complete. This will provide us the quantity which is the consequence of surface by surface calculation. 


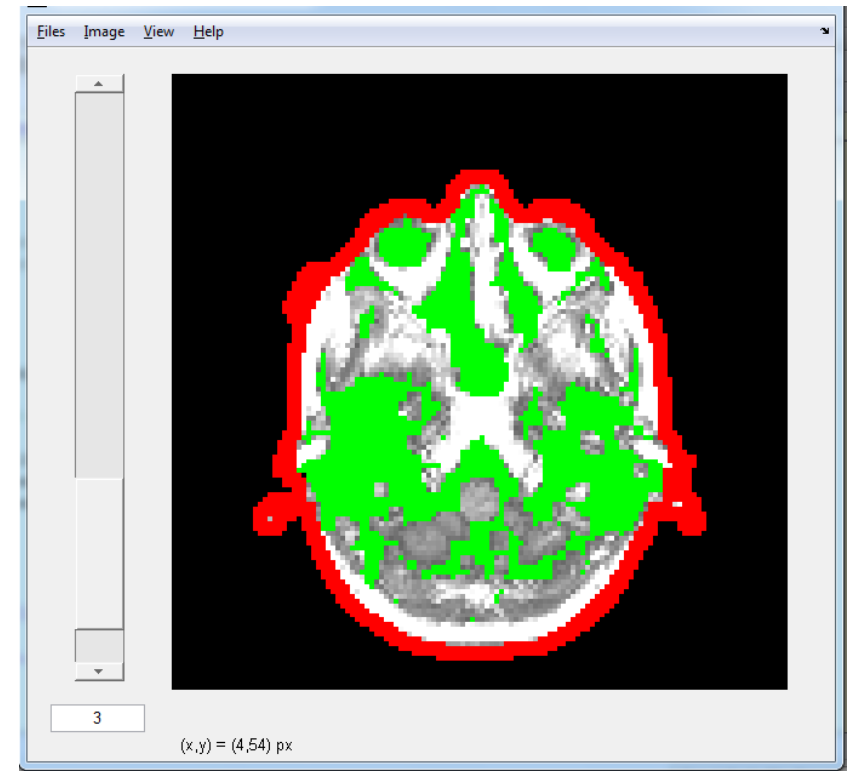

Figure 8. $X \& Y$ position MRI view.

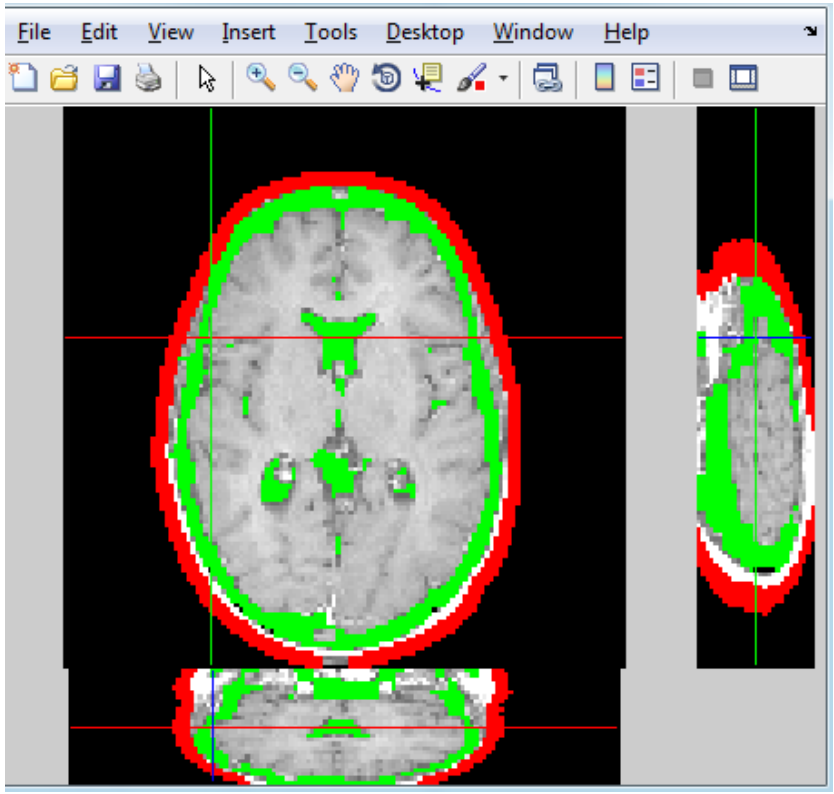

Figure 9. SBIR ortho slicer view point GUI.

\section{MRI 3D plot}

Another feature of this model is obtained by drag the mouse pointer in image GUI position. Model show allows adjust the dream property of person model or model hierarchies. By using this unit the tumor obtains is practical to the non-payment VTK of the brain replica of the slicer. This indicates the site of the tumor in the brain as in Figure 10.

One significant feature of the filtering ROI of such medical images is that those images are mono spectral: coloring and, ultimately, tissue recognition is done using a look-up table. This means that a uneven of the values may have a theatrical effect on the visualization and consequently on the understanding of the images. Median lessens the risk of misinterpretation. However, customary median filtering does not protect well the contours, and may take away small particulars which may be crucial to a physician's analysis.

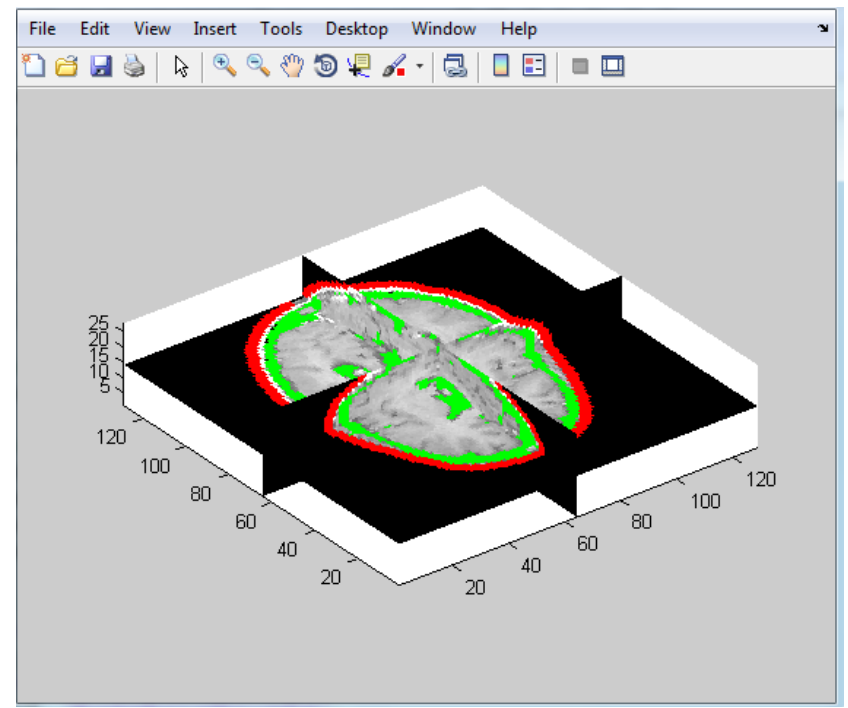

Figure 10. MRI Brain 3D plots in ROI.

\section{MRI 3D plot in different angle}

The $\mathrm{X}, \mathrm{Y}, \mathrm{Z}$ three coordinate 3D measurable angles in ROI based Slicer Image retrieval. The MRI 3D plot in different angle is shown in Figure 11.

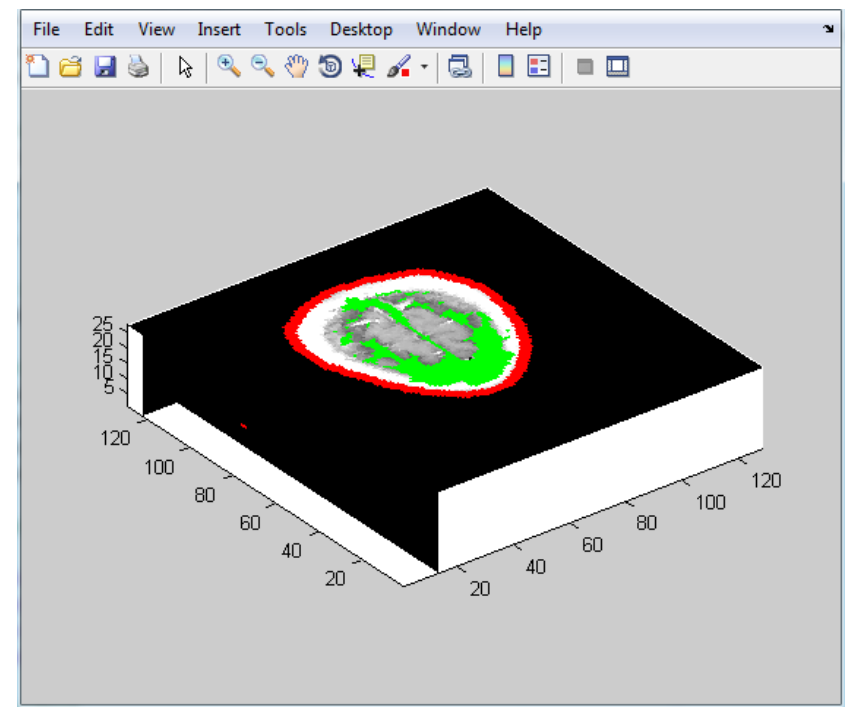

Figure 11. Different angles in $3 D$.

The proposed SBIR resolution algorithm can be applied to arbitrary 3D images or even 3D volumes of anisotropic differently dimensions. In this experiment, we apply the reconstruction algorithm to the full $3 \mathrm{D}$ MRI image volume. Figure 11 shows a volume rendering of an original image of dimensions $256 \times 256 \times 160$, at MRI widths given by $1 \times 1 \times$ $1.25 \mathrm{~mm}^{3}$.

This image is first sub sampled to half the resolution at $128 \times$ $128 \times 80\left(2 \times 2 \times 2.5 \mathrm{~mm}^{3}\right)$ super-resolved to a full isotropic $256 \times 256 \times 160$ image with $1 \times 1 \times 1 \mathrm{~mm}^{3}$ resolution. As 
expected, we can see a development in the resolution plus an increase in the detail at the same time across all $\mathrm{X}, \mathrm{Y}$, and $\mathrm{Z}$ dimensions.

In this paper, we used an anisotropic Gaussian kernel with the variances proportional to the $3 \mathrm{D}$ dimensions. Furthermore the grid dimensions for the edge-preserving up sampling.

\section{D MRI histogram analysis}

The Graphical view of Histogram approach as per input image selection the tip tag point different as per MRI image. The reason of this study was to procedure $2 \mathrm{D}$ medical data and to convert them into 3D (Figure 12). Thus, it was try to above all read the medical images, and then understand the segmentation processes on them, do the fractal geometry studies, which was the method necessary to be practical, on the medical image, and finally to present a 3D chart replica by transitory these present medical data from the march cube algorithm. In the processes in fractal stage, some arithmetical analysis of the present images was performing concerning the fractal geometry. The box including method of the fractal geometry was applied on these medical images and the region and selfsimilarity methods were used. This is the section of the medical images that was transferred to the $z$ dimension, i.e. 3D after using various boundary follow-up and similarity methods.

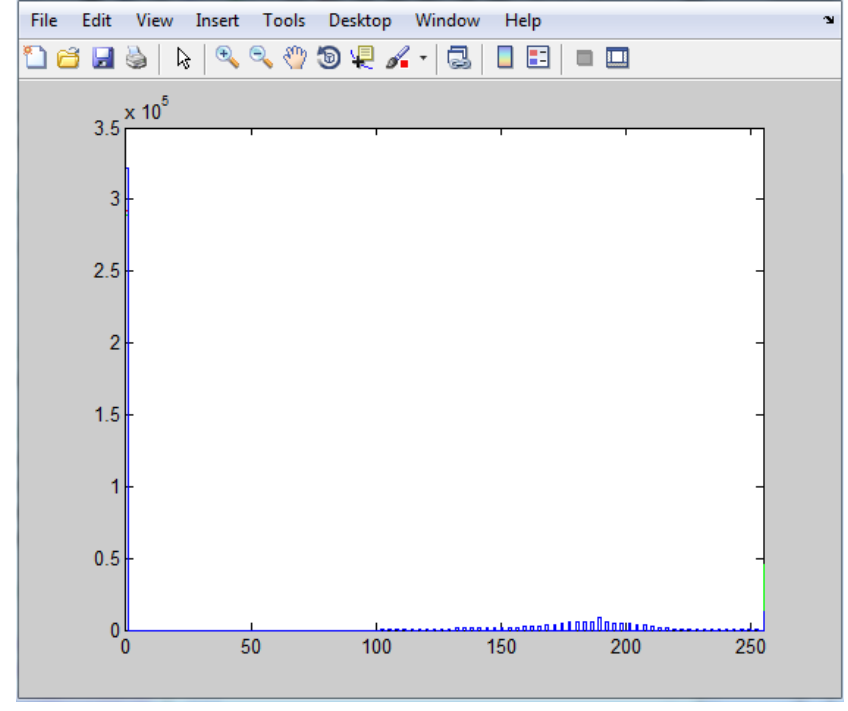

Figure 12. MRI histogram analyses in $3 D$.

\section{Comparison of the proposed method with other existing methods}

To find the effectiveness of the proposed method, the proposed SBIR is compared with other existing methods like multiplanar rendering [3], contour based surface rendering [1], marching cube algorithm [2] and improved marching cube algorithm [4]. The compassion is done in areas: demerits, demerits and performance. The comparative resulted are shown in Table 2.

Table 2. Comparative analysis of different $3 D$ reconstruction technique.

\begin{tabular}{|c|c|c|c|}
\hline Method Name & Merits & Demerits & Performance \\
\hline Multiplanar Rendering [31] & $\begin{array}{l}\text { 1. It is possible to visualize data that was } \\
\text { measured in different slices in a single } 2 D \text { image } \\
\text { 2. Speed of the method }\end{array}$ & Visualization is in $2 \mathrm{D}$ only & $\begin{array}{l}\text { Performance increases when the } \\
\text { number of iteration increases }\end{array}$ \\
\hline Contour based surface Rendering [32] & $\begin{array}{l}\text { Algorithm is efficient and it reduces the search } \\
\text { space }\end{array}$ & Ambiguities in connecting the contour & Computation cost decreases \\
\hline Marching Cube Algorithm [33] & Simple to follow the steps & $\begin{array}{l}\text { Generation of ambiguous surface and } \\
\text { huge quantities of triangular patches }\end{array}$ & $\begin{array}{l}\text { Computation is fast and results have } \\
\text { good resolution }\end{array}$ \\
\hline $\begin{array}{l}\text { Improved Marching Cube Algorithm } \\
\text { [34] }\end{array}$ & $\begin{array}{l}\text { 1. Reduces the triangular patches } \\
\text { 2. Increased speed and quality }\end{array}$ & More computations are needed & $\begin{array}{l}\text { Takes less time than standard MC } \\
\text { algorithm to finish the reconstruction } \\
\text { without compromising the quality of } \\
\text { results }\end{array}$ \\
\hline Proposed method & $\begin{array}{l}\text { 1. It is possible to visualize data that was } \\
\text { measured in different slices in a single } 2 \mathrm{D} \text { image } \\
\text { 2. Increased speed and quality } \\
\text { 3. Simple to follow the steps } \\
\text { 4. Algorithm is efficient and it reduces the search } \\
\text { space }\end{array}$ & No demerits & $\begin{array}{l}\text { Takes less time than standard } \\
\text { algorithms. It will generate the image } \\
\text { without compromising the quality of } \\
\text { results. Result with good resolution }\end{array}$ \\
\hline
\end{tabular}

\section{Conclusion}

In this research proposed a new 3D Medical Image Enhancement based on Adaptive Slicer Image retrieval technique. The project purpose of the contribution low resolution image in order to enhances the discontinuities and then uses Slicer based 3D imager viewer in GUI. This is a natural extension of parametric deformable model to in corporate entity demonstration and in sequence. The main contributions include: (1) store and retrieve the image from data base and choose the region of attention figures into vigorous contour and surface, which provide supple initialization and fast meeting, (2) the optimization 3D slicer approach view in different position is obtained productively 
from simulation tool. In general enclose work that enables very fast gradient and appearance-statistics based model deformations, predicting object region and boundary. Using various experiments on 3D medical images, virtual reality technology and modern medicine it is the multi-disciplinary research. The system is capable of using multiple imaging or multiple imaging devices for information.

\section{References}

1. Rahman MM, Bhattacharya P, Desai BC. A framework for medical image retrieval using machine learning and statistical similarity matching techniques with relevance feedback. IEEE Trans Inf Technol Biomed 2007; 11: 58-69.

2. Akakin HC, Gurcan MN. Content-based microscopic image retrieval system for multi-image queries. IEEE Transact Info Technol Biomed 2012; 16: 758-768.

3. Ramasubramanian B, Praphakar G, Murugeswari S. A novel approach for content based microscopic image retrieval system using decision tee algorithm. Int J Sci Eng Res 2013; 4: 584-588.

4. Guruvasuki R, Arasi AJP. MRI brain image retrieval using multi support vector machine classifier. Int J Adv Info Sci Technol 2013; 10: 29-36.

5. Long F, Zhang H, Feng DD. Fundamentals of ContentBased Image Retrieval. In: Feng DD, Siu WC, Zhang HJ (eds). Multimedia Information Retrieval and Management. Signals and Communication Technology 2003; Springer, Berlin, Heidelberg.

6. Yong CY, Chew KM, Mahmood NH, Ariffin I. Image processing tools package in medical imaging in MATLAB. Int J Edu Info Technol 2012.

7. Zitova B, Flusser J. Image registration methods: A survey. Image Vision Comput 2013; 21: 977-1000

8. Lofti M, Solimani A, Dargazany A, Afzal H, Bandarabadi M. Combinig wevelet transform and neural networks for image classification. IEEE , 41st Southeastern Symposium on System Theory, 2009.

9. Yavariabdi A, Bartoli A, Samir C, Artigues M, Da Ines D, Canis M. Mapping and characterizing endometrial implants by registering $2 \mathrm{D}$ transvaginal ultrasound to $3 \mathrm{D}$ pelvic magnetic resonance images. J Comput Med Imaging Graphics (CMIG) 2015; 45: 11-25.

10. Hama SM, Al-Ani MS. Medical image enhancement based on an efficient approach for adaptive anisotropic diffusion. Int J Adv Eng Technol 2013.

11. Sergamasco LCC, Nunes FLS. Content based retrieval for $3 \mathrm{~d}$ medical models: a study case using magnetic resonance imaging. University of Sao Paulo, 2012.

12. Minh VD, Lee SY. Two dimentional Weighted PCA algorithm for Face. IEEE Int Symposium Comput Intel Robot Automation 2005.

13. Kokate RM, Shandilya VK. Enhancement techniques of medical images-a review. Int J Adv Res Comput Commun Eng 2016.
14. Iakovidis D, Pelekis N, Kotsifakos E, Kopanakis I, Karanikas H, Theodoridis Y. A pattern similarity scheme for medical image retrieval. IEEE Trans Inf Technol Biomed 2009; 13: 442-450.

15. Yen WC, Chen YY, Chang YW. An enlargement method using un-decimated wavelet transform and shape function. Int Conf Intell Inf Hiding Multimedia Signal Process 2009.

16. Pourghassem H, Ghassemian H. Content-based medical image classification using a new hierarchical merging scheme. Comput Med Imag Graph 2008; 32: 651-661.

17. Xu C, Prince J. Snakes, shapes and gradient vector flow. IEEE Trans Image Process 1998; 7: 359-369.

18. Yang J, Duncan J. 3D image segmentation of deformable objects with joint shape-intensity prior models using level sets. Med Image Anal 2004; 8: 285-294.

19. Zhu S, Yuille A. Region competition: unifying snakes, region growing, and Bayes/MDL for multi-band image segmentation. IEEE Trans Pattern Anal Machine Intell 1996; 18: 884-900.

20. McLaughlin RA, Williamson JP, Phillips MJ, Armstrong JJ, Becker S, Hillman DR, Eastwood PR, Sampson DD. Applying anatomical optical coherence tomography to quantitative $3 \mathrm{~d}$ imaging of the lower airway. Optics Express 2008; 16: 17521-17529.

21. Ratan R, Sharma S, Sharma SK. Brain tumor detection based on multi-parameter MRI image analysis. ICGSTGVIP J 9: 9-17.

22. Pieper S, Lorensen B, Schroeder W, Kikinis R. The NAMICKit: ITK, VTK, Pipelines, Grids and 3D Slicer as an Open Platform for the Medical Image Computing Community. Proceedings of the3rd IEEE International Symposium on Biomedical Imaging, 2006.

23. Huang J, Kumar SR, Metra M, Zhu WJ, Zabith R. Spatial color indexing an applications. Int J Comput Vision 1999; 35: $245-268$.

24. Malladi R, Sethian J, Vemuri B. Shape modeling with front propagation: A level set approach. IEEE Trans Pattern Anal Machine Intell 1995; 17: 158-175.

25. Haney SM, Thompson PM, Cloughesy TF, Alger JR, Toga AW. Tracking tumor growth rates in patients with malignant gliomas: A test of two algorithms. AJNR Am J Neuroradiol 2001; 22: 73-82.

26. Selvaraj K. Data extraction from computer acquired images of a given 3D environment for enhanced computer vision and its applications in kinematic design of robs. J Comput Sci 2010; 6: 425-427.

27. Purschke F. VirtualReality: New methods for improving and accelerating the development process in vehicle styling and design. Comput Graphics Int 1998.

28. Mengzhou Y, Jiaoying S. 3D model reconstruction algorithm based on the texture of photos. RuanJianXueBao 2000; 11: 502-506.

29. Zhu Y, Papademetris X, Sinusas A, Duncan J. Segmentation of left ventricle from 3D cardiac MR image sequences using a subject-specific dynamical model. Comput Vision Pattern Recognit 2008. 
30. Harikrishnan S, Yogapriya J. Content based medical image retrieval using texture features. Int $\mathrm{J}$ Adv Res Technol 2012; 2: 50-54.

31. Jae-Jeong C. Efficient multidimensional volume rendering. Proceed SPIE 3658, Medical Imaging 1999.

32. Fuchs H, Kedem ZM, Uselton SP. Optimal surface reconstruction from planar contours. Communicat ACM 1977.

33. Lorensen WE, Cline HE. Marching cubes: A high resolution 3d surface construction algorithm. Comput Graphics 1987.

34. Xiao J, Yu M, Jia N. An improved MC algorithm applied in medical image reconstruction. 2008 Chinese Control and Decision Conference, Yantai, Shandong, 2008.

\section{"Correspondence to}

Padmaja Grandhe

Research Scholar

CSE Department

JNTUK

India 